\title{
Protocol
}

\section{Examining the effecting of a life-coaching intervention on breast cancer survivors' post treatment adjustment: protocol of a parallel group randomized controlled trial}

\author{
Sarkis Meterissian ${ }^{1}$, Virginia Lee ${ }^{2}$, Wing Lam Tock ${ }^{3}$, Sophie Blondin ${ }^{1}$, Christine Maheu ${ }^{3}$
}

\author{
${ }^{1}$ Breast Centre, Department of Surgery, McGill University Health Centre-Montreal, QC, Canada \\ ${ }^{2}$ Supportive and Palliative Care Programs and Services, Department of Nursing, McGill University Health Centre- \\ Montreal, QC, Canada \\ ${ }^{3}$ Ingram School of Nursing, Faculty of Medicine and Health Sciences, McGill University-Montreal, QC, Canada
}

Received: 19 August 2021

Revised: 03 September 2021

Accepted: 04 September 2021

\section{*Correspondence:}

Dr. Sarkis Meterissian,

E-mail: sarkis.meterissian@muhc.mcgill.ca

Copyright: (C) the author(s), publisher and licensee Medip Academy. This is an open-access article distributed under the terms of the Creative Commons Attribution Non-Commercial License, which permits unrestricted non-commercial use, distribution, and reproduction in any medium, provided the original work is properly cited.

\section{ABSTRACT}

Background: The growing number of breast cancer survivors in the country has warranted health interventions targeted to mitigate the bio-psychosocial impact associated with a cancer diagnosis and the toxicities of oncological treatments. Life coaching is a widely adopted intervention strategy to promote an individual's positive psychological adaptation, self-management, and self-confidence. This present study will investigate whether a life coaching intervention with group and individual coaching components can significantly improve quality of life (QoL) and post traumatic growth among breast cancer survivors.

Methods: This study is a randomized controlled trial with three parallel study arms. A prospective sample size of 120 adult breast cancer survivors will be randomized into one of the three study groups either to receive: 1) group coaching following by individual coaching sessions; 2) group coaching sessions only; or 3) routine care. The primary outcome evaluates post traumatic growth and secondary outcomes assess QoL and fear of cancer recurrence (FCR). Data collection will occur at baseline (T0) and at 3 weeks (T1), and at 18 weeks (T2). A follow-up assessment will occur at 30 weeks (T3).

Conclusions: This is the first randomized control trial to address post traumatic growth among breast cancer survivors using a life coaching intervention. If positive, the results of this study could inform intervention development that benefits the health of cancer survivors.

Trial registration: This trial is ethically approved and registered with clinical trial registry (NCT05020561).

Keywords: Breast cancer survivors, Life coaching, Post traumatic growth, QoL, FCR, Randomized controlled trial

\section{INTRODUCTION}

Advances in cancer treatment and early detection efforts have yielded encouraging results in breast cancer survivorship. The 5-year survival rate for breast cancer, the most common cancer among women in Canada, has improved markedly since the early $90 \mathrm{~s}^{1}{ }^{1}$ The growing number of breast cancer survivors in the country has warranted health interventions targeted to mitigate the biopsychosocial impact associated with a cancer diagnosis and the toxicities of anticancer treatments. ${ }^{2,3}$ Further, cancer survivors often realize that a cancer diagnosis can change them physically and psychologically, but the meaning and their perspective of life could also be changed because of this life-threatening episode. Transitioning into survivorship (i.e., the period after cancer treatment), can bring new and unexpected challenges such as FCR and decreased QoL. ${ }^{4,5}$ 
Researchers have developed a myriad of behavioural interventions that can benefit the health of cancer survivors. Health behaviour change interventions remain one of the options with the highest therapeutic value on both psychological and physical health (e.g., improved physical functioning and QoL, and mitigation of anxiety) for this patient population. ${ }^{6}$ A systematic review by Lee et al found that meaning and meaning-making can also be used as an outcome of and psychosocial adjustment to cancer. ${ }^{7}$ Life coaching is a coaching process based on meaningful personal objectives as opposed to professional objectives. Life coaching activities embed patient-centred care, health literacy, self-management support, and sustained behaviour change. The five key areas of the life coaching practice include: (1) Assessing the needs and resources of the patient, (2) Exchanging information and providing education regarding any number of client identified needs, (3) accelerating access to services and resources, (4) Providing support with a focus on patient empowerment, and (5) Building selfadvocacy competencies that aid in identifying system barriers and solutions. ${ }^{8,9}$ It is a widely adopted intervention strategy to promote positive psychological adaptation, self-management, and self-confidence in diverse settings, including education and healthcare. ${ }^{10-12}$

Few studies on breast cancer survivorship care plan have been published on the effectiveness of a life coach, a majority of the studies were descriptive rather than experimental. ${ }^{13}$ Two high quality experimental trials were identified. Kvale and colleagues tested the efficacy of the patient owned survivorship transition care for activated, empowered survivors (post-care) intervention in promoting survivor activation and self-management of survivorship health issues as participants transition from active treatment to follow-up care. ${ }^{14}$ The post-care intervention is framed on the chronic care model of Wagner and also incorporates elements of the care transitions intervention (CTI) model of Coleman. ${ }^{15,16}$ The Wagner model states that for participants to manage their transition successfully, they must set goals and establish a health plan. The CTI model tries to achieve this through self-management and increased patient activation. CTI uses a coaching model to teach transitional care to participants and assist them with setting priorities and goals. ${ }^{17}$ This randomized study had participants take part in either the above post-care intervention or usual care. The post-care intervention sessions used a coach to explore the cancer experience, identify health goals, promote self-management techniques and address barriers to goal accomplishment. The study showed a significant improvement in QoL and a significant decrease in depression, in the participants in the post-care intervention. Another approach to the post-treatment phase of care is the Extended cancer education for longerterm survivors (excels) trial. This randomized trial with 500 participants will compare: (1) Excels website, (2) Excels-health coaching, (3) Excels website and health coaching, and (4) a print booklet and measure patient outcomes such as QoL and adherence to follow-up regimens. $^{18}$
There is an increasing interest in investigating the effectiveness of health or life coaching strategies to facilitate the transition from active treatment to breast cancer survivorship with improved self-efficacy and selfmanagement and PTG, which concerns reconstructing one's life structure following traumatic events, including health issues such as cancer diagnoses. ${ }^{8-10,20,21}$ These studies recognize that successful survivorship care should transcend beyond the simple transfer of knowledge of survivorship care plans in order to produce a noticeable improvement in 'participants' transition from patient to survivor and increase their sense of self-efficacy. In this study, the life coach attempts to activate the survivors' awareness and sense of agency to engage, readjust, and manage their life in the post-treatment period.

\section{Objectives}

This present study will study the effects of a life coach to help patients with breast cancer transition from the active to the post-treatment phase. ${ }^{22,23}$ The life coaching intervention is designed to: a) ease the transition between cancer treatment and active life, and b) Allow breast cancer survivors to take control of their lives. The primary objective of this study is to determine whether individual life coaching can significantly improve PTG and QoL, and decrease FCR among women who have been recently treated with breast cancer.

\section{METHODS}

\section{Trial design and setting}

This single site study is a single blinded, randomized control trial with three parallel arms. Arm 1 participants will receive group coaching sessions followed by individual coaching sessions; arm 2 participants will receive only the group coaching sessions. Arm 3 participants will receive the routine care as provided at the McGill university health centre (MUHC) breast clinic. The routine care group (arm 3) will not be prevented from accessing available psychological resources available within or outside of MUHC, but this information will be assessed, recorded and integrated into the data analysis. Participants in arm 3 will be offered the life coaching intervention at the end of the study upon their wish. All life coaching sessions will be conducted by professional coaches certified by the International Coaching Federation (ICF). In order to implement the physical distancing measures imposed by the COVID-19 pandemic, all intervention activities will be carried out virtually via videoconferencing (e.g., Zoom).

\section{Participant's eligibility criteria}

All participants will be recruited at the MUHC breast clinic. Participants will be eligible to participate in this study if they: 1) have been diagnosed with stage I, II, or III breast cancer; 2) are female breast cancer patients over the age of $18 ; 3$ ) have completed anti-cancer treatment via surgery and/or chemotherapy and/or radiation therapy 
at the MUHC; 4) are fluent in written and spoken English or French; 5) are alert and oriented and capable of giving informed consent, and 6) have an Internet connection at home that supports participation in coaching sessions via videoconferencing. Participants will be ineligible to participate in this study if they: 1) have a recurrence of breast cancer 2) are diagnosed with a second cancer; 3) are diagnosed with stage IV breast cancer due to their worse prognosis, and 4) have an active psychiatric diagnosis that would render adherence to the study problematic.

\section{Intervention}

In this study, the group life coaching sessions will be focusing on providing material and tools that guide the participants in transition from cancer treatment to survivorship. In life coaching session one, participants will be informed about the coaching process. The life coach will help participants determine their personal objectives and indicators of success that will be explored in the subsequent sessions. The group life coaching session two aims to assess the participants' challenges or losses related to their cancer diagnosis. In group life coaching session three, the life coach will assist the participants to identify their accomplishments, challenges, needs and steps to move forward. Each group session lasts 120 minutes with a maximum of four participants in one cohort, and each group session will be completed weekly (i.e., all group coaching sessions will be completed within a three-week time frame).

In the individual life coaching sessions (i.e., life coaching sessions four to eight), the life coach will provide guidance that helps the participants to put selfmanagement tools into action. Participants will receive an intervention manual that allows them to outline their objectives and indicators of success. The life coach will also assign activities, such as self-reflection, for the participants to complete between each session. The document used in all sessions to guide the activities is named the "After cancer passport," which can be accessed in supplementary material 1 (link: https://osf.io/u6ztw/). All life coaching sessions will be conducted by certified coaches, and will be delivered virtually via videoconferencing.

\section{Hypotheses}

It is hypothesized that: 1) participants who receive both the group coaching sessions and the individualized coaching sessions will report greater PTG and QoL, and less FCR when compared to the breast cancer survivors who received only the group coaching sessions or routine care; and 2) participants who receive only the group coaching sessions will report greater PTG and QoL, and lesser FCR when compared to the participants who receive routine care.

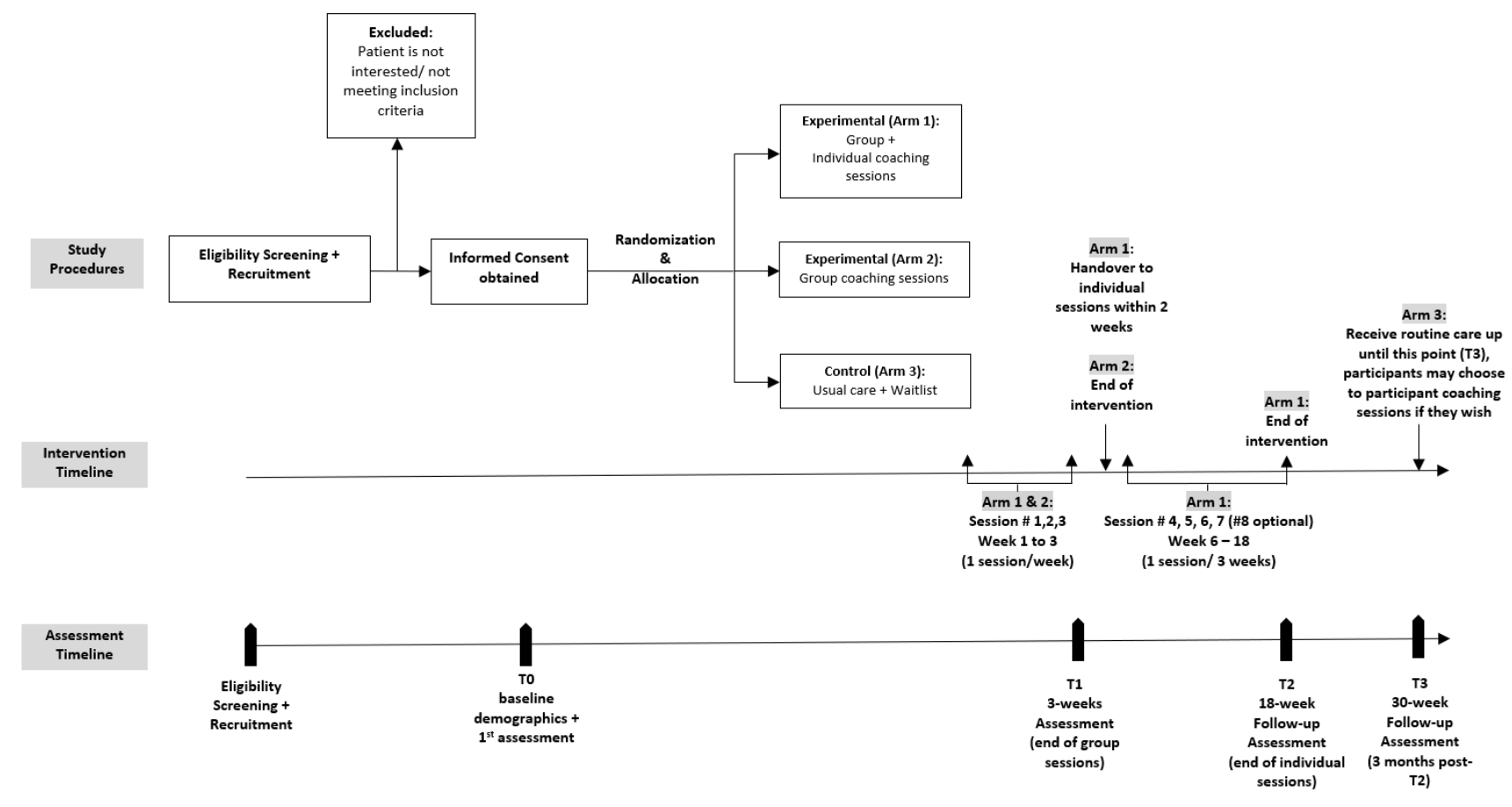

Figure 1: Study procedure flowchart.

\section{Study procedures}

The study procedure is displayed in Figure 1. Recruitment posters will be posted in MUHC breast clinic to recruit participants. Further, members of the treating team at the MUHC breast clinic (i.e., administrative staff, oncology nurse, surgical/medical/radiation therapy oncologist) will identify and seek permission from the participant before referring potentially eligible participants to the research 
assistant of this study. The research assistant will call each potential participant to explain the study in detail and obtain written informed consent from the participant with an electronic signature on the informed consent form. The participants will have access to a copy of the consent form by email or mail. A statistician outside of the research team member will be responsible for the blind allocation of the group to which a participant will belong, according to the randomization software. The consenting participants will be randomized into one of the three study arms on the basis of the computergenerated sequence of random blocks of six with an allocation ratio of $1: 1: 1$. For every two blocks of participants recruited, the 12 participants will be equally randomized into the three study arms and the intervention arms one and two will begin the life coaching group. Baseline assessment (T0) will be collected from all study participants. The group coaching sessions with four participants in each group will then be complemented within three weeks. For participants in the experimental arm one, handover to individual coaching sessions will be done within two weeks after the last group coaching session is completed (T1). Participants in arm one will receive four to five individual coaching sessions via teleconferencing that last 60 minutes each. The fifth individual session is optional and will be scheduled only if new issues had emerged at the fourth session. Participants in arm two will receive only the first three life coaching sessions (i.e., the group sessions). Participants in arm three will receive routine care by the breast clinic team for the entire course of the study. All study participants will be completing the study outcome assessments at the same time points. All individual group sessions will be expected to be completed within 15 weeks (or 18 weeks if the fifth session is required by some participants) (T2), but will allow for flexibility in session scheduling depending on the participant's availability and adherence. A 30-week follow-up (T3) assessment will be collected from all study participants.

\section{Outcome measurements}

\section{Demographics}

Demographic data will be collected from all participants at the beginning of the study. Demographic questions including age, gender, ethnicity, household income, preferred language, occupation, educational levels, and social support. One additional item will assess for potential contamination (i.e., the receipt of active intervention amongst participants in the control arm), or potential co-intervention bias (i.e., additional treatments, advice or other interventions that the participants in the experimental group have received, which could affect the outcome of interest). ${ }^{24,25}$ "Since you first received the breast cancer diagnosis, have you sought any type of supportive care services within or outside of MUHC (e.g., consultation with a psychologist, complementary therapist (yoga, meditation, art therapy), emotional support groups, etc.)? If yes, please specify the type of support." This item will be assessed at all four study time points (i.e., T0, T1, T2, T3).

\section{Primary outcome: post traumatic growth (PTG)}

The primary outcome will be assessed using the post traumatic growth inventory (PTGI). ${ }^{21}$ The PTGI consists of 21 items, the response scale is a six points Likert scale asking respondents the degree to which changes occurred in their lives as a result of the crisis, where 0 represented "I did not experience this change" and 5 represented "I experienced this change to a very great degree". The PTGI is composed of five subscales, with five items measuring the construct new possibilities, seven items measuring the construct relating to others, four items measuring the construct personal strength, three items measuring the construct appreciation of life and two items measuring the construct spiritual change. The scores are summed to provide an overall measure of PTG ranging from 0 to 105 where higher scores indicate more reported growth. ${ }^{21,26}$ The internal consistency of the inventory is $\alpha=0.90$.

\section{Secondary outcome: $Q o L$}

This secondary outcome will be assessed using the patient reported outcomes measurement information system (PROMIS) preference questionnaire (i.e., the PROPr). ${ }^{27,28}$ The PROPr is a 31 items instrument assessing eight domains (29 items) (i.e., anxiety, depression, fatigue, pain interference, physical function, sleep disturbance, ability to participate in social roles and activities, and pain intensity), and cognitive function abilities (two items). Raw scores generated for each domain are transformed into T scores; higher PROMIS T scores indicate greater endorsement of the construct being assessed. ${ }^{27}$

\section{Secondary outcome: FCR}

This secondary outcome will be assessed using the cancer worry scale (CWS). ${ }^{29}$ The CWS is a six items scale designed to measure worry about the risk of developing cancer and the impact of worry on daily functioning. The six items are rated on a four-points Likert scale ranging from one ("never") to four ("almost always"). Possible scores range from 6 to 24 with higher scores indicating more worry. This scale demonstrated good construct validity, convergent and divergent validity, and high internal consistency $(\alpha=0.90) .{ }^{29}$

\section{Data collection and management}

The data collection schedule for the study outcome measurements is shown in Table 1. At pre-intervention bassline (T0), the research assistant will be collecting the informed consent forms, as well as the following measures from each participant: baseline demographics, first set of study outcome questionnaires (i.e., PTGI, PROPr, and CWS). Following completion of the T0 data 
collection, participants will be randomly allocated to one of the three study arms. At T1 (i.e., 3 weeks post baseline, group coaching sessions completed), all participants will be completing the second set of study outcome questionnaires. At T2 (18 weeks post-baseline, individual coaching sessions completed), participants will be completing the third set of study outcome questionnaires. This will also be the first post-trial follow-up for participants in arms two and three. All participants will be completing the second post-trial follow-up at T3 (30 weeks post-baseline and three months after T2). The post-trial follow questionnaire is identical to the study outcome questionnaires (i.e., consisting of the PTGI, PROPr, and CWS). Study participants will be completing all questionnaires via Qualtrics, a secure online data collection and management platform registered with McGill university, Ingram school of nursing.

Table 1: Outcome measurement schedule.

\begin{tabular}{|c|c|c|c|c|c|}
\hline Target data & $\begin{array}{l}\text { Method of } \\
\text { assessment }\end{array}$ & $\begin{array}{l}\text { Baseline } \\
\text { (T0) }\end{array}$ & $\begin{array}{l}3 \text { weeks } \\
\text { assessment (T1) }\end{array}$ & $\begin{array}{l}18 \text { weeks } \\
\text { assessment (T2) }\end{array}$ & $\begin{array}{l}30 \text { weeks } \\
\text { assessment (T3) }\end{array}$ \\
\hline $\begin{array}{l}\text { Sample } \\
\text { demographic }\end{array}$ & $\begin{array}{l}\text { Demographic } \\
\text { questionnaire }\end{array}$ & $\mathrm{X}$ & & & \\
\hline $\begin{array}{l}\text { Potential for } \\
\text { co-intervention }\end{array}$ & One item & $X$ & $\mathrm{X}$ & $\mathrm{X}$ & $\mathrm{X}$ \\
\hline PTG & PTGI & $X$ & $X$ & $X$ & $X$ \\
\hline QoL & $\begin{array}{l}\text { PROMIS }{ }^{\circledR} \\
\text { preference (PROPr) }\end{array}$ & $\mathrm{X}$ & $\mathrm{X}$ & $\mathrm{X}$ & $\mathrm{X}$ \\
\hline FCR & CWS & $X$ & $X$ & $X$ & $X$ \\
\hline
\end{tabular}

\section{Quality management}

\section{Minimizing missing data, dropouts and attrition}

The research assistant of the study will communicate regularly with the study participants to ensure questionnaires are completed and returned as efficiently as possible. The survey will be in a secured link sent to the participants by email, they will be asked to complete the survey within a two-week timeframe. The survey is identified using unique participants identifiers, this will allow the research team to associate the participants with the questionnaires. E-mail reminders will be sent to the participants if the questionnaire was not completed and submitted after 2-weeks (note: on the Qualtrics platform, the authorized research team member will be able to see if the questionnaires are completed and submitted). We will make two contacts (i.e., sending the e-mail reminders twice within 4 weeks, once every two weeks) before considering the participant a lost to follow up. It is possible that study participants who are randomized to the control group may drop out of the study because they prefer to receive the Experimental treatment of coaching sessions. To minimize the potential of this happening, participants will be offered the coaching sessions after they complete the study (i.e., a wait-list control group). The participants can withdraw their consent or stop participating without giving any reasons and at any time. If the dropped-out participant wishes to, he/she can give a reason why they are dropping out, but they are not obliged to give a reason. Whether a response is given will have no impact on the quality of care and services they are otherwise entitled to at the MUHC or on their relationship with the researcher or clinical team.

\section{Intervention fidelity}

A fidelity checklist will be developed to monitor the extent to which our intervention is implemented and received as intended (supplementary material 2, link: https://osf.io/h5prz/). All life coaching sessions will be recorded, and a research team member will rate the activity adherence and skills performed by the life coach in the recordings against the fidelity checklist. $25 \%$ from the recordings will be randomly assessed to ensure fidelity of the intervention.

\section{Statistical analysis}

\section{Sample size calculation}

Based on data in the literature on the PTGI assuming a conservative mean difference of 10 between control and experimental groups and also assuming a conservative standard deviation of 14.86, 35 cases are required on each arm considering $5 \%$ of tolerance and $80 \%$ of statistical power. ${ }^{30}$ After allowing $10 \%$ of the loss to follow-up, a total of 120 participants or 40 participants per group are required to achieve our primary objective. Hence, 40 participants will be allocated to each of the three study arms.

\section{Data analysis}

An intention to treat analysis will be carried out. Exploratory analyses will assess the adequacy of participant characteristics to validate the balancing of the randomization process. The descriptive analysis will employ comparing each intervention arm with the control group independently, using Fisher's exact test for 
categorical and Wilcoxon rank tests for continuous outcomes to assess the associations of outcomes. Multivariate regression using logistic or multinomial methods will be used to assess the primary and secondary study outcomes. All analyses will be performed using STATA version 13.

\section{Ethical issues}

A confidential security plan aiming to minimize potential risks of confidentiality breach during the course of the study during the COVID-19 pandemic will be followed. All research staff (i.e., the research assistants and the coaches) in charge of participant recruitment, data collection, and intervention implementation will work virtually from home and/or at the MUHC/McGill offices. All intervention sessions will be implemented via videoconferencing in a private location (i.e., research 'staffs' and 'participant's home), all non-authorized personnel will be restricted from overhearing conversations (e.g., research 'staffs' spouses). Passwords will be required for all online meetings in order to restrict attendees to only the research staff and study participants. To ensure a secure work environment during remote study intervention implementation, no personally identifiable information of participants will be visible in the background or on desktop, no personal information will be used in titles or descriptions of meetings (e.g., group session meetings will be titled as "lifecoach_group_session_001_dd/mm/yy"). All data collection procedures in this study will be carried out on the Qualtrics platform, all members of the research team will acquire adequate knowledge about the use of the platform before the commencement of the study. Study participants will receive a link to complete the study survey on the Qualtrics platform during the four data collection timepoints via their personal emails. Data stored in Qualtrics will involve only the responses to the survey questions and no personal identifications. Only research staff will be authorized access to research data.

\section{DISCUSSION}

There is a need to adequately support early-stage breast cancer patients who face specific and unique challenges related to post-treatment reintegration with an uncertain future in a meaningful way. Comparing to the services provided by the traditional psychologist and psychiatric professionals, whose aims usually involve resolving traumatic events that happened in the past of a person's life, life coaching practice focuses on leading an individual to move forward in life by adopting a variety of resources, tools, and activities. Further, the services provided by psychologists and psychiatrists might be associated with stigma. Life coaching is a promising complementary practice that might be incorporated in routine cancer survivorship care as post-treatment support.

\section{CONCLUSION}

This proposed study is novel and timely as healthcare teams strive to improve the transition to survivorship. This study explores the effectiveness of the life coaching practice in assisting cancer patients to adapt to the new normal post-treatment. The intervention provided in the study will help breast cancer survivors to ground themselves to their reality, and the phone coaching will assist survivors to actualize their next steps. Further, the life coach facilitates survivors' expression of their needs and teaches the survivors to proactively engage with their healthcare team. This study will be the first to address PTG among breast cancer survivors using life coaching interventions with the ultimate objective of improving the QoL of breast cancer survivors.

Funding: Quebec Breast Cancer Foundation, Breast Cancer Support and Education Fund

Conflict of interest: None declared

Ethical approval: Research Institute of the McGill University Health Centre: Research Ethics Board (REB) on 2021/07/26. This trial is registered on clinicaltirla.gov NCT05020561.

\section{REFERENCES}

1. Canadian Cancer Society. Canadian cancer statistics 2020: Cancer-specific statistics. Available at https://www.cancer.ca/en/cancer-information/cancer101/cancer-statistics-at-a-glance/?region=on.

Accessed 25 August 2021.

2. Nekhlyudov L, Mollica MA, Jacobsen PB, Mayer DK, Shulman LN, Geiger AM. Developing a quality of cancer survivorship care framework: Implications for clinical care, research, and policy. J Natl Cancer Inst. 2019;111(11):1120-30.

3. Takahashi M. Health promotion for cancer survivors: New paradigm beyond prevention and treatment. In: Muto T, Nakahara T eds. Asian Perspectives and Evidence on Health Promotion and Education. Tokyo: Springer Japan. 2011;78-86.

4. Sun H, Yang Y, Zhang J. Fear of cancer recurrence, anxiety and depressive symptoms in adolescent and young adult cancer patients. Neuropsychiatr Dis Treat. 2019;15:857-65.

5. Sodergren SC, Husson O, Rohde GE. A life put on pause: An exploration of the health-related quality of life issues relevant to adolescents and young adults with cancer. J Adolesc Young Adult Oncol. 2018;7(4):453-64.

6. Boland L, Bennett K, Connolly D. Self-management interventions for cancer survivors: A systematic review. Support Care Cancer. 2018;26(5):1585-95.

7. Lee V, Cohen S, Edgar L, Laizner A, Gagnon A. Meaning-making intervention during breast or colorectal cancer treatment improves self-esteem, optimism, and self-efficacy. Soc Sci Med. 2006;62:3133-45. 
8. Eagen L, Levesque J. Transforming community cancer care: The Ottawa regional cancer foundation's cancer coaching practice. University Ottawa J Med. 2017;7:1.

9. Post L, Liefbroer AI. Reducing distress in cancer patients-A preliminary evaluation of short- term coaching by expert volunteers. Psycho-Oncology. 2019;28(8):1762-6.

10. Ammentorp J, Uhrenfeldt L, Angel F, Ehrensvärd M, Carlsen EB, Kofoed PE. Can life coaching improve health outcomes? A systematic review of intervention studies. BMC Health Serv Res. 2013;13(1):428.

11. Olsen JM, Nesbitt BJ. Health coaching to improve healthy lifestyle behaviors: An integrative review. Am J Health Promot. 2010;25(1):e1-12.

12. Wagland R, Fenlon D, Tarrant R, Howard-Jones G, Richardson A. Rebuilding self-confidence after cancer: a feasibility study of life-coaching. Support Care Cancer. 2015;23(3):651-9.

13. Timmermann C, Ammentorp J, Birkelund R. Person- centred communication with cancer survivors: Exploring the meaning of follow- up coaching conversations. Scand J Caring Sci. 2021.

14. Kvale EA, Huang CS, Meneses KM. Patientcentered support in the survivorship care transition: Outcomes from the patient-owned survivorship care plan intervention. Cancer. 2016;122(20):3232-42.

15. Wagner EH. Chronic disease management: What will it take to improve care for chronic illness? Eff Clin Pract. 1998;1(1):2-4.

16. Coleman EA, Parry C, Chalmers S, Min S-J. The care transitions intervention. Arch Intern Med. 2006;166(17):1822.

17. Coleman EA, Smith JD, Frank JC, Min SJ, Parry C, Kramer AM. Preparing patients and caregivers to participate in care delivered across settings: The care transitions intervention. $\mathrm{J}$ Am Geriatr Soc. 2004;52(11):1817-25.

18. Davis SN, O'Malley DM, Bator A. Rationale and design of extended cancer education for longer term survivors (EXCELS): A randomized control trial of 'high touch' vs. 'high tech' cancer survivorship selfmanagement tools in primary care. BMC Cancer. 2019;19:1.

19. Barakat S, Boehmer K, Abdelrahim M, et al. Does health coaching grow capacity in cancer survivors? A systematic review. Popul Health Manag. 2017;21(1):63-81.
20. Bellizzi KM, Blank TO. Predicting posttraumatic growth in breast cancer survivors. Health Psychol. 2006;25(1):47.

21. Tedeschi RG, Calhoun LG. The posttraumatic growth inventory: Measuring the positive legacy of trauma. J Trauma Stress. 1996;9(3):455-71.

22. Ganz PA. Late effects of cancer and its treatment. Semin Oncol Nurs. 2001;17(4):241-8.

23. Ganz PA. Quality of care and cancer survivorship: The challenge of implementing the institute of medicine recommendations. J Oncol Pract. 2009;5(3):101-5.

24. Magill N, Knight R, McCrone P, Ismail K, Landau $\mathrm{S}$. A scoping review of the problems and solutions associated with contamination in trials of complex interventions in mental health. BMC Me Res Methodol. 2019;19:1.

25. Aggarwal R, Ranganathan P. Study designs: Part 5interventional studies (II). Perspect Clin Res. 2019;10(4):183-6.

26. Holtmaat K, Van der Spek N, Cuijpers P, Leemans CR, Verdonck-de Leeuw IM. Posttraumatic growth among head and neck cancer survivors with psychological distress. Psycho-Oncol. 2017;26(1):96-101.

27. Dewitt B, Jalal H, Hanmer J. Computing PROPr utility scores for PROMIS $₫$ profile instruments. Value in Health. 2020;23(3):370-8.

28. Hays RD, Spritzer KL, Schalet BD, Cella D. PROMIS®-29 v2.0 profile physical and mental health summary scores. Qual Life Res. 2018;27(7):1885-91.

29. Custers JAE, Kwakkenbos L, Van De Wal M, Prins JB, Thewes B. Re-validation and screening capacity of the 6-item version of the Cancer Worry Scale. Psycho-Oncol. 2018;27(11):2609-15.

30. Brix SA, Bidstrup PE, Christensen J. Post-traumatic growth among elderly women with breast cancer compared to breast cancer-free women. Acta Oncol. 2013;52(2):345-54.

Cite this article as: Meterissian $\mathrm{S}$, Lee V, Tock WL, Blondin S, Maheu C. Examining the effecting of a life-coaching intervention on breast cancer survivors' post treatment adjustment: protocol of a parallel group randomized controlled trial. Int J Clin Trials 2021;8(4):278-84. 